\title{
SURFACE AREA. II
}

BY

\section{HERBERT FEDERER}

1. Introduction. All definitions of $\$ 2$ of our preceding paper, Surface Area. I( $\left.{ }^{1}\right)$, are again in force.

Throughout the present paper we fix two positive integers $m \leqq n$. We are interested in the validity of the formula

$$
\int N(f, T, y) d \Phi y=\int_{T} J f(x) d x
$$

where $f$ is a function on $E_{m}$ to $E_{n}$ and $T \subset E_{m}$. The left-hand member of this equation is the integral, relative to our $m$-dimensional surface measure over $E_{n}$, of the multiplicity of $f$ on $T$. We believe that this integral appropriately measures the area of that part of the surface $f$ which corresponds to $T$. The integrand of the right-hand member is the Jacobian associated with $f$ by means of its approximate differential.

Our main results are contained in the Theorems 5.1, 5.2, 6.1 and 7.2. The last of these, which is a specialization of the preceding theory, yields new information about Dini derivatives.

In case $m=2$ it follows from the work of Radó, McShane, and Morrey( $\left.{ }^{2}\right)$ that the right-hand member of the identity frequently equals the Lebesgue surface area of $f$.

If $f$ is univalent, the left-hand integral is the $\Phi$ measure of the image of $T$ under $f$.

2. Definition of the measure $\Phi$.

2.1 Definition. We say $P$ is a projecting function (on $E_{n}$ to $E_{m}$ ) if and only if there is an orthogonal transformation $R$ on $E_{n}$ to $E_{n}$ such that the relations

$$
R(x)=y \text { and } P(x)=\left(y_{1}, y_{2}, \cdots, y_{m}\right)
$$

are equivalent whenever $x$ and $y$ are points in $E_{n}$.

For $S \subset E_{n}$ we define $\gamma(S)$ to be the supremum of numbers of the form $\left|P^{*}(S)\right|$ where $P$ is a projecting function $\left({ }^{3}\right)$.

Presented to the Society, October 30, 1943; received by the editors September 16, 1943.

(1) Trans. Amer. Math. Soc. vol. 55 (1944) pp. 420-437. We hereafter refer to this paper as SAI.

(2) T. Rad6, Über das Flächenmass rektifizierbarer Flächen, Math. Ann. vol. 100, p. 445; On the derivative of the Lebesgue area of continuous surfaces, Fund. Math. vol. 30, p. 34.

E. J. McShane, Integrals over surfaces in parametric form, Ann. of Math. vol. 34, p. 815.

C. B. Morrey, A class of representations of manifolds, Amer. J. Math. vol. 55, p. 683.

$\left.{ }^{3}\right)\left|P^{*}(S)\right|$ is the $m$-dimensional Lebesgue measure of $P^{*}(S)$. 
Whenever $S \subset E_{n}$ and $r>0$, we denote by $\gamma_{r}(S)$ the infimum of numbers of the form

$$
\sum_{T \in F} \gamma(T)
$$

where $F$ is a countable family of open connected subsets of $E_{n}$, each of diameter less than $r$, such that $S \subset \sigma(F)$.

We now define the function $\Phi$ on the set of all subsets of $E_{n}$ by the relation

$$
\Phi(S)=\lim _{r \rightarrow 0+} \gamma_{r}(S) .
$$

This limit exists because $\gamma_{r}(S)$ is monotone in $r$ for each fixed set $S$.

2.2 Remark. If $m=2$ and $n=3$, then the functions $\gamma, \gamma_{r}, \Phi$ of Definition 2.1 are identical with the functions bearing the same names in SA I.

In fact the reader will easily convince himself that the functions $P_{c}$ introduced in $\$ 3$ of SA I are projection functions in the sense of 2.1 of this paper; and each of our present projection functions is of the form $R: P_{c}$ where $R$ is an orthogonal transformation of $E_{2}$. Using the invariance of plane Lebesgue measure under such transformations it may be seen that the old and new meanings of $\gamma$ are identical; consequently the same is true for $\gamma_{r}$ and $\Phi$.

2.3 Remark. Theorems 3.4, 3.5, 3.6, 4:4 of SA I are true in our present general setting with $P_{c}$ replaced by any projecting function $P$. The proofs are exactly the same as before.

2.4 Remark. If $m=n$, then $\Phi$ is $m$-dimensional Lebesgue measure.

2.5 Remark. If $m=1$, then $\Phi$ is Carathéodory linear measure over $E_{n}$. In fact $\gamma(T)=\operatorname{diam} T$ for every connected set $T \subset E_{n}$.

3. Further definitions.

3.1 Definition. We write $\operatorname{det} A$ for the determinant of a square matrix $A$.

Now suppose $p$ and $q$ are positive integers.

A function $L$ on $E_{p}$ to $E_{q}$ is said to be linear if and only if $L$ is continuous and

$$
L(x+y)=L(x)+L(y) \text { for } x \in E_{p}, y \in E_{p} .
$$

We make no distinction between $L$ and its matrix. The $j$ th column $(j=1,2, \cdots, p)$ of $L$ is a point of $E_{q}$ and denoted by $L^{j}$. We further define the norm of $L$ by the relation

$$
\|L\|=\sup _{|x|=1}|L(x)| .
$$

If $p \leqq q$, then

$$
\Delta(L)=\left\{\sum_{x \in S}\left(\operatorname{det} \dot{M}_{x}\right)^{2}\right\}^{1 / 2}
$$

where $x \in S$ if and only if $x$ is a set of $p$ integers between 1 and $q$; and $M_{x}$ is the minor of $L$ which is made up from the $p$ rows whose indices are elements of $x$. 
3.2 Definition. Suppose $g$ is a numerically valued function on $E_{p}$ and $x \in E_{p}$. Then we define

$$
\lim \sup _{z \rightarrow x} \operatorname{ap} g(z)
$$

as the infimum of numbers of the form

$$
\lim _{z \rightarrow x, z \in A} \sup _{A} g(z)
$$

where $A$ is a Lebesgue measurable subset of $E_{p}$ with density 1 at $x$.

3.3 Definition. Suppose $f$ is a function on $E_{p}$ to $E_{q}$.

We say $f$ is differentiable at $x$ if and only if there is a linear function $L$ on $E_{p}$ to $E_{q}$ such that

$$
\limsup _{z \rightarrow x} \frac{|f(z)-f(x)-L(z-x)|}{|z-x|}=0 .
$$

The (unique) linear function occurring in this definition is called the differential of $f$ at $x$.

We say $f$ is approximately differentiable at $x$ if and only if there is a linear function $L$ on $E_{p}$ to $E_{q}$ such that

$$
\lim \sup _{z \rightarrow x} \operatorname{ap} \frac{|f(z)-f(x)-L(z-x)|}{|z-x|}=0 .
$$

The (unique) linear function occurring in this definition is called the $a p$ proximate differential of $f$ at $x$.

If $p \leqq q$ then we define the function $J f$ as follows: The domain of $J f$ is the set of all points of approximate differentiability of $f$ and for each such point $x$ we let

$$
J f(x)=\Delta(L)
$$

where $L$ is the approximate differential of $f$ at $x$.

3.4 Remark. Suppose $f$ is a function on $E_{m}$ to $E_{n}$.

It may interest the reader, although we shall not use it, that in case $m=2$ our definitions imply

$$
J f=\left(E G-F^{2}\right)^{1 / 2}
$$

where the right-hand member has its classical meaning.

Furthermore in case $m=n$ our $J f(x)$ equals the absolute value of the classical Jacobian determinant of $f$ at $x$.

Similarly in case $m=1$ our $J f(x)$ is the absolute value of the approximate derivative of $f$ at $x$.

3.5 Remark. If $f$ is a univalent differentiable function on $E_{m}$ to $E_{n}$, then $f$ transforms $E_{m}$ into a Riemannian manifold $R_{m}$. Fix a point $x \in E_{m}$ and let $g$ be the matrix of the fundamental differential quadratic form associated with the point $f(x) \in R_{m}$ and the coordinate system defined by $f$. Denoting the 
differential of $f$ at $x$ by $L$ and its conjugate by $\bar{L}$, we have $g=\bar{L}: L$ and see from the proof of Lemma 4.2 below that $(\operatorname{det} g)^{1 / 2}=J f(x)$.

No use is made of this fact in this paper.

\section{Lipschitzian surfaces.}

4.1 LeMma. If $f$ is a function on $T \subset E_{m}$ to $E_{n}$ and $M$ is a number such that then

$$
|f(z)-f(x)| \leqq M|z-x| \text { whenever } x \in T, z \in T
$$

Proof. Let $r>0$.

$$
\Phi\left[f^{*}(T)\right] \leqq(5 M)^{m}|T| \text {. }
$$

Let $S$ be an open set for which $T \subset S$ and $|S| \leqq|T|+r$. Whenever $x \in T$, $\rho>0$ and

$$
\alpha=E_{m} \underset{z}{E}[|z-x| \leqq \rho]
$$

we denote

$$
\tilde{\alpha}=E_{m} \underset{z}{E}[|z-x|<5 \rho], \quad \bar{\alpha}=E_{n} \underset{y}{E}[|y-f(x)|<5 M \rho]
$$

and readily check that

$$
f^{*}(T \tilde{\alpha}) \subset \bar{\alpha}, \quad \gamma(\bar{\alpha})=M^{m}|\tilde{\alpha}|=(5 M)^{m}|\alpha| .
$$

Now let $F$ be the family of all closed spheres $\alpha$ with center in $T$ and such that $\alpha \subset S$, diam $\bar{\alpha}<r$. Clearly $F$ covers $T$ and we use a well known covering theorem( $\left.{ }^{4}\right)$ to select such a disjointed subfamily $G$ of $F$ that

Hence $f^{*}(T) \subset \sum_{\alpha \in G} \bar{\alpha}$ and

$$
T \subset \sum_{\alpha \in G} \tilde{\alpha} \text {. }
$$

$$
\gamma_{r}\left[f^{*}(T)\right] \leqq \sum_{\alpha \in G} \gamma(\bar{\alpha})=(5 M)^{m} \sum_{\alpha \in G}|\alpha| \leqq(5 M)^{m}|S| \leqq(5 M)^{m}(|T|+r) .
$$

Let $r \rightarrow 0$.

4.2 LemMa. If $L$ is a linear function on $E_{m}$ to $E_{n}$ and $R$ is an orthogonal transformation on $E_{n}$ to $E_{n}$, then $\Delta(R: L)=\Delta(L)$.

Proof. For each $n \times m$ matrix $A$ we denote its conjugate $m \times n$ matrix by $\bar{A}$, that is $\bar{A}_{\mathfrak{t}}^{j}=A_{j}^{i}$; hence the Cauchy-Binet theorem( ${ }^{(5)}$ implies

$$
[\Delta(A)]^{2}=\operatorname{det}(\bar{A}: A) \text {. }
$$

From this we infer

$$
\begin{aligned}
& {[\Delta(R: L)]^{2}=\operatorname{det}[(\overline{R: L}):(R: L)]=\operatorname{det}[(\bar{L}: \bar{R}):(R: L)]} \\
& \quad=\operatorname{det}[\bar{L}:(\bar{R}: R): L]=\operatorname{det}[(\bar{L}: L)]=[\Delta(L)]^{2} .
\end{aligned}
$$

(4) A. P. Morse, A theory of covering and differentiation, Trans. Amer. Math. Soc. vol. 55 (1944) pp. 205-235, Theorem 3.5.

(5) A. C. Aitken, Determinants and matrices, Edinburgh, 1942, p. 86. 
4.3 LeMma. If $f$ is a function on $E_{m}$ to $E_{n} ; M$ is a number such that

$$
|f(z)-f(x)| \leqq M|z-x| \text { for } z \in E_{m}, x \in E_{m} ;
$$

$A$ is a Lebesgue measurable subset of $E_{m}$; $f$ is differentiable at each point of $A$ and

then

$$
0<\lambda<J f(x)<\mu<\infty \text { for } x \in A \text {; }
$$

$$
\lambda|A| \leqq \int N(f, A, y) d \Phi y \leqq \mu|A| .
$$

Proof. Denoting the family of all Lebesgue measurable subsets of $A$ by $K$, we infer from 4.1 that

$$
f^{*}(X) \text { is } \Phi \text { measurable for } X \in K .
$$

For $x \in A$ let $D f(x)$ be the differential of $f$ at $x$. For $k=1,2,3, \ldots$ we define

$$
\eta_{k}(x)=\sup _{0<|z-x|<x^{-1}} \frac{|f(z)-f(x)-[D f(x)](z-x)|}{|z-x|}
$$

and are told by 3.3 that

$$
\eta_{k}(x) \rightarrow 0 \text { as } k \rightarrow \infty \text { for each } x \in A .
$$

Helped by Egoroff's theorem we next select such disjoint closed subsets $B_{1}, B_{2}, B_{3}, \cdots$ of $A$ that

$$
|A-B|=0, \text { where } B=\sum_{s=1}^{\infty} B_{s}
$$

and

$$
\eta_{k}(x) \rightarrow 0 \text {, uniformly for } x \in B_{s} \text {, as } k \rightarrow \infty
$$

for each positive integer $s$. Thus $f$ is uniformly differentiable on $B_{\text {. }}$ Using the standard theorem on uniform convergence we also note that the partial derivatives $D_{1} f, D_{2} f, \cdots, D_{m} f$ are continuous relative to $B_{3}$. In the terminology of Hassler Whitney $\left(^{6}\right)$ all this implies that $f$ is of class $C^{\prime}$ in $B_{\text {s }}$ in terms of the functions $f$ and $D_{1} f, D_{2} f, \cdots, D_{m} f$ for each positive integer $s$.

Now Whitney's extension theorem $\left({ }^{7}\right)$ gives us such functions $g_{1}, g_{2}, g_{3}, \ldots$ with continuous partial derivatives on $E_{m}$ to $E_{n}$ that

$$
g_{s}(x)=f(x) \text { and } D_{j} g_{s}(x)=D_{j} f(x)
$$

for $x \in B_{s} ; s=1,2,3, \cdots ; j=1,2, \cdots, m$. Since

$$
\left|D_{j} g_{s}(x)\right|=\left|D_{j} f(x)\right| \leqq M \text { for } x \in B_{s}
$$

( $\left.{ }^{6}\right)$ H. Whitney, Analytic extensions of differentiable functions defined in closed sets, Trans. Amer. Math. Soc. vol. 36 (1934) p. 64.

(7) Loc. cit. $\left(^{(}\right)$, p. 69, Lemma 2. 
and $D_{j} g_{2}$ is continuous, we can select open sets $Q_{1}, Q_{2}, Q_{3}, \cdots$ such that $B . \subset Q, C E_{m}$ and

$$
\left|g_{s}(x)-g_{s}(z)\right| \leqq 2 M|x-z| \text { whenever } x \in Q_{s}, z \in Q_{s} .
$$

The remainder of the proof is divided into three parts.

Part 1. If $s$ is a positive integer, $x \in B$, and $r>0$, then there is a family $F(x)$ of closed subsets of $Q$, such that

$$
0=\inf _{\alpha \in F(x)} \operatorname{diam} \alpha ; \quad 0<\inf _{\alpha \in F(x)} \frac{|\alpha|}{(\operatorname{diam} \alpha)^{m}} ;
$$

$\alpha \in F(x)$ implies $x \in \alpha, \lambda|\alpha|<\Phi\left[g_{s}^{*}(\alpha)\right]$ and associated with $\alpha$ is an open sphere $\bar{\alpha} \subset E_{n}$ for which $g_{s}^{*}(\alpha) \subset \bar{\alpha}$, diam $\bar{\alpha}<r$ and $\gamma(\bar{\alpha})<\mu|\alpha|$.

Proof. Abbreviate $L=D f(x)$. Select an orthogonal transformation $R$ on $E_{n}$ to $E_{n}$ such that

$$
R\left(L^{i}\right) \in E_{n} \underset{y}{E}\left[y_{i}=0 \text { for } i=m+1, m+2, \cdots, n\right]
$$

for $j=1,2, \cdots, m$; and let $P$ be the projecting function such that the relations

$$
R(y)=z \text { and } P(x)=\left(z_{1}, z_{2}, \cdots, z_{m}\right)
$$

are equivalent whenever $y$ and $z$ are points of $E_{n}$. Take

$$
p=P: g_{s}, \quad T=P: L,
$$

and note that $L$ is the differential of $g_{\varepsilon}$ at $x$. Thus $T$ is the differential of $p$ at $x$ and from (3) and Lemma 4.2 we infer

$$
J p(x)=|\operatorname{det} T|=\Delta(T)=\Delta(P: L)=\Delta(R: L)=\Delta(L)=J f(x)>0 .
$$

Using (3) again we verify

$$
|T(w)|=|R[L(w)]|=|L(w)| \text { for } w \in E_{m} .
$$

By virtue of (4) there exists an inverse $U$ of $T$.

Now choose $\epsilon$ so that $0<\epsilon\|U\|<1$ and

$$
\lambda<J f(x)(1-\epsilon\|U\|)^{m}<J f(x)(1+\epsilon\|U\|)^{m}<\mu
$$

and then select such a positive number $\delta$ that

$$
2 \delta(1+\epsilon\|U\|)<r\|U\|
$$

and

(8) $\left|g_{8}(z)-g_{s}(x)-L(z-x)\right|<\epsilon|z-x|$ whenever $0<|z-x|<\delta$.

Next the classical theorem on continuously differentiable functions with nonvanishing Jacobian yields in view of (4) a number $t$ such that $0<t<\delta$ and 


$$
p^{*}(S) \text { is an open subset of } E_{m} \text {, }
$$

where

$$
S=E_{m} \underset{z}{E}[|z-x|<t] \subset Q_{s} .
$$

Now a typical set $\alpha \in F(x)$ is constructed as follows:

Choose a number $\rho$ for which $0<\rho\|U\|<t$ and

$$
w \in p^{*}(S) \text { whenever }|w-p(x)|<(1-\epsilon\|U\|) \rho .
$$

Corresponding to this number $\rho$ let

$$
\begin{aligned}
& \alpha=E_{m} \underset{z}{E}[|T(z-x)| \leqq \rho], \\
& \bar{\alpha}=E_{n} \underset{y}{E}\left[\left|y-g_{8}(x)\right|<(1+\epsilon\|U\|) \rho\right] .
\end{aligned}
$$

Clearly $x \in \alpha, \alpha$ is closed, $\bar{\alpha}$ is an open sphere of $E_{n}$, and diam $\bar{\alpha}=2 \rho(1+\epsilon\|U\|)$ $<2 \delta\|U\|^{-1}(1+\epsilon\|U\|)<r$ by virtue of (7). A moment's thought convinces us that

$$
\begin{gathered}
|\alpha| \cdot|\operatorname{det} T|=\left|E_{m} \underset{z}{E}[|z| \leqq \rho]\right| \\
\gamma(\bar{\alpha})=(1+\epsilon\|U\|)^{m}\left|E_{m} \underset{z}{E}[|z| \leqq \rho]\right|
\end{gathered}
$$

Next we check

$$
\begin{aligned}
|z-x|= & |U[T(z-x)]| \leqq\|U\|_{\rho}<t<\delta \text { for } z \in \alpha ; \\
& \alpha \subset S \subset Q_{z} \text { and } \operatorname{diam} \alpha<2\|U\|_{\rho} .
\end{aligned}
$$

Evidently $g_{\odot}(x) \in \bar{\alpha}$ and the relation $x \neq z \in \alpha$ implies via (12), (8), (5), (12) that

$$
\begin{aligned}
\left|g_{s}(z)-g_{s}(x)\right| & <|L(z-x)|+\epsilon|z-x| \leqq|T(z-x)|+\epsilon\|U\| \rho \\
& \leqq \rho(1+\epsilon\|U\|),
\end{aligned}
$$

hence $g_{s}(z) \in \bar{\alpha}$. Accordingly

$$
g_{s}^{*}(\alpha) \subset \bar{\alpha} .
$$

Now let

$$
C=E_{m} \underset{w}{E}[|w-p(x)|<(1-\epsilon\|U\|) \rho] .
$$

If $w \in C$ we use (9) to pick $z \in S$ with $p(z)=w$. Since $t<\delta$ we may use (8) and (12) to check

$$
\begin{aligned}
|p(z)-p(x)-T(z-x)| & =\left|P\left[g_{s}(z)\right]-P\left[g_{s}(x)\right]-P[L(z-x)]\right| \\
& =\left|P\left[g_{s}(z)-g_{s}(x)-L(z-x)\right]\right| \\
& \leqq\left|g_{s}(z)-g_{s}(x)-L(z-x)\right| \\
& \leqq \epsilon|z-x| \leqq \epsilon|| U|| \cdot|T(z-x)|,
\end{aligned}
$$




$$
\begin{aligned}
(1-\epsilon\|U\|) \rho & \geqq|p(z)-p(x)| \\
& \geqq|T(z-x)|-\epsilon\|U\| \cdot|T(z-x)| \\
& =(1-\epsilon\|U\|) \cdot|T(z-x)|,
\end{aligned}
$$

hence $\rho \geqq|T(z-x)|$ and $z \in \alpha, w \in p^{*}(\alpha)$. Thus

$$
C \subset p^{*}(\alpha) \text {. }
$$

From Theorem 3.6 of SA I (see Remark 2.3 of this paper) and the relations (15), (10), (4), (6) we infer

$$
\begin{aligned}
\Phi\left[g_{s}^{*}(\alpha)\right] & \geqq\left|P\left[g_{s}^{*}(\alpha)\right]\right|=\left|p^{*}(\alpha)\right| \geqq|C| \\
& =(1-\epsilon|| U \|)^{m}\left|E_{m} \underset{z}{E}[|z| \leqq \rho]\right|=(1-\epsilon|| U||)^{m}|\alpha| \cdot|\operatorname{det} T| \\
& =(1-\epsilon\|U\|)^{m} J f(x)|\alpha|>\lambda|\alpha| ; \\
& \lambda|\alpha|<\Phi\left[g_{s}^{*}(\alpha)\right] .
\end{aligned}
$$

From (11), (10), (4), (6) we similarly obtain

$$
\gamma(\bar{\alpha})<\mu|\alpha| \text {. }
$$

At last we use (10) and (13) to check that

$$
\frac{|\alpha|}{(\operatorname{diam} \alpha)^{m}} \geqq \frac{\left|E_{m} \underset{z}{E}[|z| \leqq \rho]\right|}{|\operatorname{det} T| \cdot(2|| U \| \mid)^{m}}=\frac{\left|E_{m} E_{z}[|z| \leqq 1]\right|}{|\operatorname{det} T| \cdot(2 \| U||)^{m}}
$$

and observe that the right-hand member of the last equation is a positive number independent of $\rho$ and $\alpha$.

We combine the last remark with the relations (13), (16), (14), (17) to complete the proof of Part 1.

Part 2. If $X \in K$ and $X \subset B_{s}$, then

$$
\lambda|X| \leqq V_{K}(f, X, \Phi) \text { and } \Phi\left[f^{*}(X)\right] \leqq \mu|X| \text {. }
$$

Proof. Let $r>0$.

Choose an open set $S$ such that $X \subset S$ and $|S-X| \leqq r$.

For $x \in X$ define $F(x)$ as in Part 1 and let

$$
\begin{aligned}
G(x) & =F(x) \underset{\alpha}{E}[\alpha \subset S], \\
U & =\sum_{x \in X} G(x) .
\end{aligned}
$$

The existence of a countable disjointed family $H \subset U$ for which $|X-\sigma(H)|$ $=0$ may be deduced from the classical Vitali covering theorem $\left({ }^{8}\right)$ or from the

(8) S. Saks, Theory of the integral, Warsaw, 1937, p. 109. 
fact that $G$ is a diametrically regular blanket $\left({ }^{\circ}\right)$. Now use Part 1 , Lemma 4.1 and (2) to obtain

$$
\begin{aligned}
\gamma_{r}\left[f^{*}(X)\right] & =\gamma_{r}\left[g_{s}^{*}(X)\right] \leqq \gamma_{r}\left\{g_{s}^{*}[\sigma(H)]\right\}+\gamma_{r}\left\{g_{s}^{*}[X-\sigma(H)]\right\} \\
& \leqq \sum_{\alpha \in H} \gamma(\bar{\alpha})+\Phi\left\{g_{s}^{*}[X-\sigma(H)]\right\} \\
& \leqq \sum_{\alpha \in H} \mu|\alpha|+(10 M)^{m}|X-\sigma(H)| \\
& \leqq \dot{\mu}|S| \leqq \mu(|X|+r)^{\prime} \\
\lambda|X| & \leqq \lambda \sum_{\alpha \in H}|\alpha| \leqq \sum_{\alpha \in H} \Phi\left[g_{s}^{*}(\alpha)\right] \leqq \sum_{\alpha \in H} \Phi\left[g_{s}^{*}(\alpha X)\right]+\Phi\left[g_{s}^{*}(\alpha-X)\right] \\
& \leqq \sum_{\alpha \in H} \Phi\left[f^{*}(\alpha X)\right]+(10 M)^{m}|\alpha-X| \\
& \leqq V_{K}(f, X, \Phi)+(10 M)^{m}|S-X| \leqq V_{K}(f, X, \Phi)+(10 M)^{m_{r}} .
\end{aligned}
$$

Thus

$$
\lambda|X| \leqq V_{K}(f, X, \Phi)+(10 M)^{m_{r}} \text { and } \gamma_{r}\left[f^{*}(X)\right] \leqq \mu(|X|+r)
$$

for every $r>0$. Let $r \rightarrow 0$.

Part 3. $\lambda|A| \leqq \int N(f, A, y) d \Phi y \leqq \mu|A|$.

Proof. We use (1), Lemma 4.1 and Theorem 4.1 of SA I to check:

$$
\begin{aligned}
0= & |A-B|=\Phi\left[f^{*}(A-B)\right], \\
& \sum_{s=1}^{\infty}\left|B_{s}\right|=|B|=|A|, \\
\sum_{s=1}^{\infty} V_{K}\left(f, B_{s}, \Phi\right)= & \sum_{s=1}^{\infty} \int N\left(f, B_{s}, y\right) d \Phi y=\int \sum_{s=1}^{\infty} N\left(f, B_{s}, y\right) d \Phi y \\
= & \int N(f, B, y) d \Phi y=\int N(f, A, y) d \Phi y .
\end{aligned}
$$

We shall accordingly complete the proof by showing that

$$
\lambda\left|B_{s}\right| \leqq V_{K}\left(f, B_{s}, \Phi\right) \leqq \mu\left|B_{s}\right| \text { for } s=1,2,3, \cdots .
$$

Fixing a positive integer $s$, we see that the first inequality in (18) follows immediately from Part 2. If, on the other hand, $G \subset K$ and $G$ is a partition of $B_{s}$, then

$$
\sum_{s \in G} \Phi\left[f^{*}(S)\right] \leqq \mu \sum_{s \in G}|S|=\mu\left|B_{s}\right|
$$

by virtue of Part 2. Considering the arbitrary nature of $G$, we are convinced of the second inequality in (18).

4.4 LEMMA. If $f$ is a function on $E_{m}$ to $E_{n} ; M$ is a number such that

$$
|f(z)-f(x)| \leqq M|z-x| \text { for } z \in E_{m}, x \in E_{m} \text {; }
$$

(9) Loc. cit. ( $\left.{ }^{(}\right)$, Definition 6.5 and Theorem 6.12 . 
$A$ is a Lebesgue measurable subset of $E_{m} ; f$ is differentiable at each point of $A$ and $J f(x)=0$ for $x \in A$; then $\Phi\left[f^{*}(A)\right]=0$.

Proof. We assume, without loss of generality, that $|A|<\infty$ and divide the remainder of the proof into two parts.

Part 1. If $x \in A$ and $r>0$, then there is a closed sphere $\alpha \subset E_{m}$ and an open connected set $\bar{\alpha} \subset E_{n}$ such that $x \in \alpha, \operatorname{diam} \alpha<r, \operatorname{diam} \bar{\alpha}<r, f^{*}(\alpha) \subset \bar{\alpha}$, $\gamma(\bar{\alpha}) \leqq r|\alpha|$.

Proof. Let $L$ be the differential of $f$ at $x$ and let $h$ be the function such that

Choose $\epsilon$ so that

$$
h(z)=f(x)+L(z-x) .
$$

$$
0<2 \epsilon\{\|L\|+\epsilon\}^{m-1}\left|E_{m-1} \underset{z}{E}[|z| \leqq 1]\right| \leqq r\left|E_{m} \underset{w}{E}[|w| \leqq 1]\right|,
$$

select $\rho$ so that $0<2 \rho\{\|L\|+\epsilon+1\}<r$ and $|f(z)-h(z)|<\epsilon|z-x|$ whenever $0<|z-x| \leqq \rho$.

Denoting by $d(y, Y)$ the distance of the point $y$ from the set $Y$, we define

$$
\alpha=E_{m} \underset{z}{E}[|z-x| \leqq \rho], \quad \bar{\alpha}=E_{n} \underset{y}{E}\left[d\left\{y, h^{*}(\alpha)\right\}<\epsilon \rho\right] .
$$

Of all the properties required for $\alpha$ and $\bar{\alpha}$, only the last is not quite obviously satisfied. In order to prove it, let $P$ be any projection function on $E_{n}$ to $E_{m}$. Denote

$$
Q=E_{m} \underset{w}{E}\left[w_{m}=0\right] \text {. }
$$

Since $\Delta(L)=J f(x)=0$, the points $L^{1}, L^{2}, \cdots, L^{m}$ are linearly dependent; hence the same is true of the points $P\left(L^{1}\right), \cdots, P\left(L^{m}\right)$. We may therefore select a distance preserving transformation $T$ on $E_{m}$ to $E_{m}$ such that

$$
[T: P: h]^{*}(\alpha) \subset Q \text {. }
$$

Let

$$
\begin{aligned}
& B=Q \underset{w}{E}[|w-[T: P: h](x)| \leqq\|L\| \rho], \\
& C=E_{m} \underset{w}{E}\left[|w-[T: P: h](x)|<\{\|L\|+\epsilon\}_{\rho} ;\left|w_{m}\right|<\epsilon \rho\right]
\end{aligned}
$$

and successively check the relations

$$
\begin{aligned}
h^{*}(\alpha) & \subset E_{n} \underset{\nu}{E}[|y-h(x)| \leqq\|L\| \rho], \quad[T: P: h]^{*}(\alpha) \subset B, \\
{[T: P]^{*}(\bar{\alpha}) } & \subset E_{m} \underset{w}{E}\left[d\left\{w,[T: P: h]^{*}(\alpha)\right\}<\epsilon \rho\right] \subset E_{m} \underset{w}{E}[d(w, B)<\epsilon \rho] \subset C . \\
\left|P^{*}(\bar{\alpha})\right| & =\left|[T: P]^{*}(\bar{\alpha})\right| \leqq|C| \\
& =2 \epsilon \rho\{\|L\|+\epsilon\}^{m-1} \rho^{m-1}\left|E_{m-1} \underset{z}{E}[|z| \leqq 1]\right| \\
& =2 \epsilon\{\|L\|+\epsilon\}^{m-1} \frac{\left|E_{m-1} \underset{z}{E}[|z| \leqq 1]\right|}{\left|E_{m} \underset{w}{E}[|w| \leqq 1]\right|}|\alpha| \leqq r|\alpha| .
\end{aligned}
$$


Thus $\left|P^{*}(\bar{\alpha})\right| \leqq r|\alpha|$ and from the arbitrary nature of $P$ we infer $\gamma(\bar{\alpha}) \leqq r|\alpha|$. Part 2. $\Phi\left[f^{*}(A)\right]=0$.

Proof. Let $r>0$.

Choose an open set $S$ such that $A \subset S \subset E_{m},|S|<|A|+r$. Let $F$ be the family of all closed spheres $\alpha \subset S$ which satisfy all the conditions of Part 1. Clearly $F$ covers $A$ in the sense of Vitali and there is a countable disjointed subfamily $G$ of $F$ such that $|A-\sigma(G)|=0$. Use Part 1 and 4.1 to infer

$$
\begin{aligned}
\gamma_{r}\left[f^{*}(A)\right] & \leqq \gamma_{r}\left\{f^{*}[\sigma(G)]\right\}+\gamma_{r}\left\{f^{*}[A-\sigma(G)]\right\} \\
& \leqq \sum_{\alpha \in G} \gamma(\bar{\alpha})+\Phi\left\{f^{*}[A-\sigma(G)]\right\} \leqq r \sum_{\alpha \in G}|\alpha|+(5 M)^{m}|A-\sigma(G)| \\
& \leqq r|S|<r\{|A|+r\} \\
\gamma_{r}\left[f^{*}(A)\right] & <r\{|A|+r\} .
\end{aligned}
$$

Let $r \rightarrow 0$.

4.5 TheOREM. If $f$ is a function on $E_{m}$ to $E_{n} ; M$ is a number such that

$$
|f(z)-f(x)| \leqq M|z-x| \text { for } z \in E_{m}, x \in E_{m}
$$

$T$ is a Lebesgue measurable subset of $E_{m}$; then

$$
\int N(f, T, y) d \Phi y=\int_{T} J f(x) d x .
$$

Proof. Let $A$ be the set of those points of $T$ at which $f$ is not differentiable. From Rademacher's theorem $\left({ }^{10}\right)$ we know that $|A|=0$. Hence $\Phi\left[f^{*}(A)\right]=0$ by 4.1. Denote

$$
B=(T-\dot{A}) \underset{x}{E}[J f(x)=0], \quad C=(T-A) \underset{x}{E}[J f(x)>0],
$$

and observe that the theorem follows immediately from the following

Statement. If $t>1$, then

$$
t^{-1} \int_{T} J f(x) d x \leqq \int N(f, T, y) d \Phi y \leqq t \int_{T} J f(x) d x .
$$

Proof. Let $\alpha$ be the set of all integers. For $j \in \alpha$ let

$$
C_{i}=C \underset{x}{E}\left[t^{i}<J f(x) \leqq t^{j+1}\right]
$$

and infer from 4.3 , taking $\lambda=t^{i}$ and letting $\mu \rightarrow t^{i+1}+$, that

$$
t^{i}\left|C_{j}\right| \leqq \int N\left(f, C_{j}, y\right) d \Phi y \leqq t^{i+1}\left|C_{j}\right|
$$

$\left({ }^{10}\right)$ H. Rademacher, Über partielle und totale Differenzierbarkeit, I, Math. Ann. vol. 79 (1919). 
because $C_{j}$ is a Borel set. Note that

and accordingly

$$
T=A+B+\sum_{j \in \alpha} C_{j}
$$

$$
\begin{aligned}
t^{-1} \int_{T} J f(x) d x & =t^{-1} \int_{A} J f(x) d x+t^{-1} \int_{B} J f(x) d x+\sum_{j \in \alpha} t^{-1} \int_{C_{j}} J f(x) d x \\
& \leqq \sum_{j \in t_{\alpha}} t^{j}\left|C_{j}\right| \leqq \sum_{j \in \in_{\alpha}} \int N\left(f, C_{j}, y\right) d \Phi y=\int \sum_{j \in \alpha} N\left(f, C_{j}, y\right) d \Phi y \\
& =\int N(f, C, y) d \Phi y=\sum_{j \in \alpha} \int N\left(f, C_{j}, y\right) d \Phi y \leqq \sum_{j \in \alpha} t^{j+1}\left|C_{j}\right| \\
& \leqq \sum_{j \in \alpha} t \int_{C_{j}} J f(x) d x \leqq t \int_{T} J f(x) d x ; \\
t^{-1} \int_{T} J f(x) d x & \leqq \int N(f, C, y) d \Phi y \leqq t \int_{T} J f(x) d x .
\end{aligned}
$$

But $\Phi\left[f^{*}(A+B)\right] \leqq \Phi\left[f^{*}(A)\right]+\Phi\left[f^{*}(B)\right]=0$ in view of our remark at the beginning of the proof and of Lemma 4.4. Thus

$$
N(f, C, y)=N(f, T, y)
$$

for $\Phi$ almost all $y$ in $E_{n}$ and we complete the proof by substitution in the last integral inequality.

\section{Approximately differentiable surfaces.}

5.1 Theorem. If $f$ is a Lebesgue measurable function on $E_{m}$ to $E_{n}, T$ is a Lebesgue measurable subset of $E_{m}$ and if there are sets $T_{1}, T_{2}, T_{3}, \cdots$ and numbers $M_{1}, M_{2}, M_{3}, \cdots$ such that

and

$$
T=\sum_{j=1}^{\infty} T_{j}
$$

$$
|f(z)-f(x)| \leqq M_{j}|z-x| \text { whenever } z \in T_{j}, x \in T_{j}
$$

then

$$
\int N(f, T, y) d \Phi y=\int_{T} J f(x) d x .
$$

Proof. Let $C$ be the set of points of approximate continuity of $f$. For each positive integer $j$ let $D_{j}$ be the set of points of density of $T_{j}$ and define

$$
S_{i}=T C D_{i}, \quad A_{j}=S_{j}-\sum_{i=1}^{j-1} S_{i}
$$

We note that $S_{j}$ is a Lebesgue measurable set with 


$$
\begin{aligned}
\left|T_{i}-S_{i}\right| & =\left|T_{i}-T C D_{i}\right|=\left|T_{i}-C D_{i}\right| \\
& \leqq\left|T_{i}-C\right|+\left|T_{i}-D_{i}\right|=0, \\
\left|T_{i}-S_{j}\right| & =0 \text { for } j=1,2,3, \cdots .
\end{aligned}
$$

The remainder of the proof is divided into four parts.

Part 1. If $x \in S_{j}, z \in S_{j}$, then $|f(z)-f(x)| \leqq M_{j}|z-x|$.

Proof. Select a measurable set $A$ with density 1 at $x$ such that $f$ is continuous relative to $A$ at $x$. Since $A$ is measurable it is easily seen(11) that $x$ is a point of density of the set $A T_{j}$, even though $T_{j}$ may be nonmeasurable. Hence there are points $u^{1}, u^{2}, u^{3}, \cdots$ such that

$$
\lim _{k \rightarrow \infty} u^{k}=x, \quad u^{k} \in A T_{j} \text { for } k=1,2,3, \cdots .
$$

It follows that

$$
\lim _{k \rightarrow \infty} f\left(u^{k}\right)=f(x), \quad u^{k} \in T_{j} \quad \text { for } \quad k=1,2,3, \cdots .
$$

Applying the same reasoning to $z$ we find points $v^{1}, v^{2}, v^{3}, \cdots$ for which

$$
\lim _{k \rightarrow \infty} v^{k}=z, \quad \lim _{k \rightarrow \infty} f\left(v^{k}\right)=f(z), \quad v^{k} \in T_{j} \quad \text { for } \quad k=1,2,3, \cdots .
$$

Accordingly

$$
\begin{aligned}
|f(z)-f(x)| & =\left|\lim _{\boldsymbol{k} \rightarrow \infty} f\left(\boldsymbol{v}^{k}\right)-\lim _{\boldsymbol{k} \rightarrow \infty} f\left(\boldsymbol{u}^{k}\right)\right|=\lim _{\boldsymbol{k} \rightarrow \infty}\left|f\left(\boldsymbol{v}^{k}\right)-f\left(\boldsymbol{u}^{k}\right)\right| \\
& \leqq M_{i} \limsup _{\boldsymbol{z} \rightarrow \infty}\left|\boldsymbol{v}^{k}-\boldsymbol{u}^{k}\right|=M_{i}|z-x| .
\end{aligned}
$$

Part 2. If $j$ is a positive integer, then

$$
\int N\left(f, A_{j}, y\right) d \Phi y=\int_{A_{j}} J f(x) d x .
$$

Proof. In view of Part 1 there exists( $\left.{ }^{12}\right)$ a function $g$ on $E_{m}$ to $E_{n}$ such that $g$ satisfies a Lipschitz condition on $E_{m}$ and

$$
g(x)=f(x) \text { for } x \in S_{j} .
$$

It follows from Theorem 4.5 that

$$
\int N\left(g, A_{j}, y\right) d \Phi y=\int_{A_{j}} J g(x) d x .
$$

(i1) The relation $|X A|+\left|X T_{i}\right|=\left|X\left(A+T_{j}\right)\right|+\left|X A T_{i}\right|$ holds for every $X \subset E_{m}$. See C. Caratheodory, Vorlesungen ibber reelle Funktionen, Leipzig, 1927, p. 252.

(19) H. Whitney, Analytic extensions of differentiable functions defined in closed sets, Trans. Amer. Math. Soc. vol. 36 (1934). Apply the formula given in the last footnote on page 63 to each of the $n$ coordinate functions. 
But $A_{j} \subset S_{j}$; hence (2) implies

$$
N\left(g, A_{j}, y\right)=N\left(f, A_{j}, y\right) \text { for } y \in E_{n} .
$$

Moreover at each point of density of $S_{j}$ at which $g$ is differentiable, the differential of $g$ is the approximate differential of $f$. Accordingly

$$
J g(x)=J f(x) \text { for almost all } x \text { in } S_{j} .
$$

Substitute (4) and (5) in (3) to establish Part 2.

Part 3. If $j$ is a positive integer, then $\Phi\left[f^{*}\left(T_{j}-S_{j}\right)\right]=0$.

Proof. Remember that $f$ satisfies a Lipschitz condition on $T_{j}$ and apply (1) and 4.1.

Part 4. $\int N(f, T, y) d \Phi y=\int_{T} J f(x) d x$.

Proof. Let $S=\sum_{j=1}^{\infty} S_{j}$; note that $A_{1}, A_{2}, A_{3}, \cdots$ are disjoint and

$$
S=\sum_{j=1}^{\infty} A_{j}, \quad(T-S) \subset \sum_{j=1}^{\infty}\left(T_{j}-S_{j}\right)
$$

Hence (1) and Part 3 imply

$$
\begin{aligned}
|T-S| & =\Phi\left[f^{*}(T-S)\right]=0, \\
N(f, T, y) & =N(f, S, y) \quad \text { for } \Phi \text { almost all } y \text { in } E_{n} .
\end{aligned}
$$

We now use (7), Part 2, (6), (8) to conclude

$$
\begin{aligned}
\int_{T} J f(x) d x & =\int_{S} J f(x) d x=\sum_{j=1}^{\infty} \int_{A_{j}} J f(x) d x \\
& =\sum_{j=1}^{\infty} \int N\left(f, A_{j}, y\right) d \Phi y=\int \sum_{j=1}^{\infty} N\left(f, A_{j}, y\right) d \Phi y \\
& =\int N(f, S, y) d \Phi y=\int N(f, T, y) d \Phi y .
\end{aligned}
$$

5.2 THEOREM. If $f$ is a Lebesgue measurable function on $E_{m}$ to $E_{n}, T$ is a Lebesgue measurable subset of $E_{m}$ and

then

$$
\lim _{z \rightarrow x} \operatorname{ap} \frac{|f(z)-f(x)|}{|z-x|}<\infty \quad \text { for every } \quad x \in T,
$$

Proof. Let

$$
\int N(f, T, y) d \Phi y=\int_{T} J f(x) d x .
$$

$$
\begin{aligned}
& K_{0}=E_{m} \underset{w}{E}[|w| \leqq 1], \\
& K_{1}=E_{m} \underset{w}{E}[|w-(1,0, \cdots, 0)| \leqq 1]
\end{aligned}
$$


and denote

$$
\alpha=\frac{\left|K_{0} K_{1}\right|}{\left|K_{0}\right|+\left|K_{1}\right|} .
$$

Since $K_{0} K_{1}$ has interior points, we know that $\alpha>0$.

For each positive integer $j$, we let $T_{j}$ be the set of all points $x$ for which $x \in T,|f(x)| \leqq j$, and $0<r \leqq j^{-1}$ implies

$$
\begin{aligned}
\mid \underset{w}{E}[|w-x| & \leqq r,|f(w)-f(x)| \leqq j|w-x|] \mid \\
& >(1-\alpha)|\underset{w}{E}[|w-x| \leqq r]| .
\end{aligned}
$$

Evidently

$$
T=\sum_{j=1}^{\infty} T_{j}
$$

and use of 5.1 will complete the proof as soon as we verify the following

Statement. If $x \in T_{j}, z \in T_{j}$, then

$$
|f(z)-f(x)| \leqq 2 j^{2}|z-x| .
$$

Proof. If $|z-x|>j^{-1}$, we have $|f(z)-f(x)| \leqq 2 j<2 j^{2}|z-x|$. We henceforth assume $|z-x|=r \leqq j^{-1}$ and define

$$
C_{0}=\underset{w}{E}[|w-x| \leqq r], \quad C_{1}=\underset{w}{E}[|w-z| \leqq r]
$$

note that

and let

$$
\alpha=\frac{\left|C_{0} C_{1}\right|}{\left|C_{0}\right|+\left|C_{1}\right|}
$$

$$
\begin{aligned}
& B_{0}=C_{0} \underset{w}{E}[|f(w)-f(x)| \leqq j|w-x|], \\
& B_{1}=C_{1} \underset{w}{E}[|f(w)-f(z)| \leqq j|w-z|] .
\end{aligned}
$$

Since $f$ is a measurable function we know that $B_{0}$ and $B_{1}$ are measurable sets and

$$
\begin{aligned}
\left|B_{0} B_{1}\right| & =\left|B_{0}\right|+\left|B_{1}\right|-\left|B_{0}+B_{1}\right| \\
& >(1-\alpha)\left|C_{0}\right|+(1-\alpha)\left|C_{1}\right|-\left|C_{0}+C_{1}\right| \\
& =(1-\alpha)\left\{\left|C_{0}\right|+\left|C_{1}\right|\right\}-\left\{\left|C_{0}\right|+\left|C_{1}\right|-\left|C_{0} C_{1}\right|\right\} \\
& =\left|C_{0} C_{1}\right|-\alpha\left\{\left|C_{0}\right|+\left|C_{1}\right|\right\}=0 .
\end{aligned}
$$

Thus $\left|B_{0} B_{1}\right|>0$ and we can pick a point $w \in B_{0} B_{1}$. Then

$$
\begin{aligned}
|f(z)-f(x)| & \leqq|f(z)-f(w)|+|f(w)-f(x)| \leqq j|z-w|+j|z-x| \\
& =2 j r=2 j|z-x| \leqq 2 j^{2}|z-x| .
\end{aligned}
$$

5.3 TheOREM. If $f$ is a continuous function on $E_{m}$ to $E_{n}, T$ is an analytic 
subset of $E_{m}$ and the approximate partial derivatives of $f$ exist (finite) at almost all points of $T$, then

$$
\int_{T} J f(x) d x \leqq \int N(f, T, y) d \Phi y .
$$

Proof. Let $S$ be the subset of $T$ on which $f$ is approximately differentiable. From Stephanoff's theorem $\left({ }^{13}\right)$ we see that $|T-S|=0$ and combine this with 5.2 to infer

$$
\int_{T} J f(x) d x=\int_{S} J f(x) d x=\int N(f, S, y) d \Phi y .
$$

But $N(f, S, y) \leqq N(f, T, y)$ for $y \in E_{n}$ and completion of the proof depends merely on showing that the last expression is $\Phi$ measurable. For this purpose we use Theorem 4.1 of SA I, taking $F=$ the family of all analytic subsets of $T$.

5.4 Remark. Suppose $\int_{T} J f(x) d x<\infty$ in addition to the hypotheses of 5.3. Then equality holds in the conclusion of that theorem if and only if

$$
\Phi\left[f^{*}(X)\right]=0 \text { whenever } X \subset T,|X|=0 .
$$

\section{Two-dimensional surfaces in $\boldsymbol{n}$-dimensional space.}

6.1 Theorem. If $f$ is a Lebesgue measurable function on $E_{2}$ to $E_{n}(2 \leqq n)$; $T$ is a Lebesgue measurable subset of $E_{2}$; corresponding to each $x \in T$ there are three distinct points $\bar{x}^{1}, \bar{x}^{2}, \bar{x}^{3}$ of $E_{2}$ such that $\left|\bar{x}^{i}\right|=1$ and

then

$$
\limsup _{t \rightarrow 0+}\left|\frac{f\left(x+t \bar{x}^{j}\right)-f(x)}{t}\right|<\infty \text { for } j=1,2,3
$$

$$
\int N(f, T, y) d \Phi y=\int_{T} J f(x) d x .
$$

Proof. Let $C=E_{2} E_{z}[|z|=1]$.

Let $W$ be the set of all matrices whose columns are three distinct points of $C$. For each $x \in T$, the points $\bar{x}^{1}, \bar{x}^{2}, \bar{x}^{3}$ are evidently the columns of the matrix $\bar{x} \in W$. Each matrix $w \in W$ determines a subdivision of $C$ into three arcs as follows: $C_{1}(w)$ leads from $w^{2}$ to $w^{3} ; C_{2}(w)$ from $w^{3}$ to $w^{1} ; C_{3}(w)$ from $w^{1}$ to $w^{2}$. Further we denote by $\delta(w)$ the smallest of the distances from $w^{j}$ to $C_{j}(w)$, where $j=1,2,3$, and observe that $\delta(w)>0$ for $w \in W$.

Now let $D$ be a countable subset of $C$ which is dense in $C$ and define

$$
Z=W \underset{w}{E}\left[w^{i} \in D \text { for } j=1,2,3\right] \text {. }
$$

We write $a \cdot b=a_{1} b_{1}+a_{2} b_{2}$ for $a \in E_{2}, b \in E_{2}$ and note that $\left|\bar{x}^{i} \cdot \bar{x}^{j}\right|<1$ whenever $x \in T ; i=1,2,3 ; j=1,2,3 ; i \neq j$.

(13) Loc. cit. $\left({ }^{7}\right)$, p. 300 . The extension to $m$ dimensions offers no difficulty. 
For each positive integer $k$ and each matrix $w \in Z$, we let $T_{k}^{w}$ be the set of all points $x$ for which:

$$
\begin{gathered}
x \in T ; \quad|f(x)| \leqq k ; \quad \delta(\bar{x})>4 k^{-1} . \\
\left|f\left(x+t \bar{x}^{j}\right)-f(x)\right| \leqq k t \text { whenever } \quad 0 \leqq t \leqq 2 k^{-1}, j=1,2,3 . \\
1-x^{i} \cdot x^{j}>3 k^{-1} \text { whenever } i=1,2,3 ; j=1,2,3 ; i \neq j . \\
\left|\bar{x}^{j}-w^{j}\right| \leqq k^{-1} \text { for } j=1,2,3 .
\end{gathered}
$$

Since $Z$ is countable and

$$
T=\sum_{k=1}^{\infty} \sum_{w \in z} T_{k}^{w}
$$

our theorem is a consequence of 5.1 and the following

Statement. If $x \in T_{k}^{w 0}$ and $z \in T_{k}^{w 0}$, then

$$
|f(z)-f(x)| \leqq 2 k^{3}|z-x| \text {. }
$$

Proof. Let $r=k^{-1}$. In case $|z-x| \geqq r^{2}$, we have

$$
|f(z)-f(x)| \leqq 2 k=2 k^{3} r^{2} \leqq 2 k^{3}|z-x|
$$

and we henceforth assume

(1)

$$
|z-x|<r^{2}
$$

We define

$$
\begin{aligned}
& K=\underset{u}{E}[|u-x|=r], \\
& S_{j}=\underset{u}{E}\left[|u-x| \leqq r, \frac{u-x}{|u-x|} \in C_{j}(\bar{x})\right], \quad K_{j}=K S_{j} \quad \text { for } \quad j=1,2,3 \text {; }
\end{aligned}
$$

and let $L_{j}$ be the line segment joining $x$ and $x+r x^{j}$. Since (1) implies $z \in \sum_{j=1}^{3} S_{j}$, we may, without loss of generality, assume $z \in S_{8}$.

The half ray issuing from $z$ with direction $\bar{z}^{3}$ intersects the boundary of $S_{3}$ in a point $u$; that is, a number $\lambda \geqq 0$ can be found such that

$$
z+\lambda \bar{z}^{3}=u \in\left(L_{1}+L_{2}+K_{3}\right) .
$$

We have $\lambda=\left|\lambda \bar{z}^{3}\right|=|u-z| \leqq 2 r$ since $z$ and $u$ are inside $K$; hence

$$
0 \leqq \lambda \leqq 2 r .
$$

We shall prove next (by contradiction) that

$$
u \in L_{1}+L_{2} \text {. }
$$

By virtue of (2), the denial of (4) implies $u \in K_{3}$. From the definition of $K_{3}$ we infer

$$
|u-x|=r, \quad(u-x) / r \in C_{3}(x),
$$


and use the definitions of $\delta(\bar{x})$ and $T_{k}^{20}$ as well as the relations (2), (1) to obtain

$$
\begin{aligned}
\delta(\bar{x}) & \leqq\left|(u-x) / r-\bar{x}^{3}\right|=k\left|z+\lambda \bar{z}^{3}-x-r \bar{x}^{3}\right| \\
& \leqq k\left\{|z-x|+\left|\lambda \bar{z}^{3}-r \bar{z}^{3}\right|+\left|r \bar{z}^{3}-r w^{3}\right|+\left|r w^{3}-r \bar{x}^{3}\right|\right\} \\
& <k\left\{r^{2}+|\lambda-r|+r^{2}+r^{2}\right\}=3 r+k|\lambda-r| \\
& =3 r+k|| u-z|-| u-x|| \leqq 3 r+k|x-z|<3 r+r<\delta(\bar{x}) ;
\end{aligned}
$$

hence $\delta(\bar{x})<\delta(\bar{x})$, which is false. Thus we have verified (4) and we may, without loss of generality, assume

$$
u \in L_{1} \text {. }
$$

We infer the existence of a number $\mu$ such that

$$
0 \leqq \mu \leqq r, \quad x+\mu \bar{x}^{1}=u .
$$

Use (2), (5) and the definition of $T_{k}^{20}$ to compute

$$
\begin{aligned}
z-x & =\mu \bar{x}^{1}-\lambda \bar{z}^{3}, \quad(z-x) \cdot\left(\bar{x}^{1}-\bar{z}^{3}\right)=(\mu+\lambda)\left(1-\bar{z}^{3} \cdot \bar{x}^{1}\right), \\
1-\bar{z}^{3} \cdot \bar{x}^{1} & =1-\bar{x}^{3} \cdot \bar{x}^{1}+\left(\bar{x}^{3}-\bar{z}^{3}\right) \cdot \bar{x}^{1} \geqq 3 r-\left|\bar{x}^{3}-\bar{z}^{3}\right| \geqq r, \\
\lambda+\mu & \leqq \frac{|z-x| \cdot\left|\bar{x}^{1}-\bar{z}^{3}\right|}{1-\bar{z}^{3} \cdot \bar{x}^{1}} \leqq(2 / r)|z-x| ; \mu+\lambda \leqq 2 k|z-x| .
\end{aligned}
$$

The last relation combines with (2), (5), (3) and the definition of $T_{k}^{20}$ to give us the result:

$$
\begin{aligned}
|f(z)-f(x)| & \leqq\left|f(z)-f\left(z+\lambda \bar{z}^{3}\right)\right|+\left|f\left(x+\mu \bar{x}^{1}\right)-f(x)\right| \\
& \leqq k \lambda+k \mu=k(\lambda+\mu) \leqq 2 k^{2}|z-x| \leqq 2 k^{3}|z-x| .
\end{aligned}
$$

6.2 Remark. It is easy to show by an example that the three directions associated with each point in 6.1 cannot be replaced by two directions.

6.3 Theorem. If the Lebesgue measurable function $f$ on $E_{2}$ to $E_{n}(2 \leqq n)$ has finite partial derivatives at each point of the Lebesgue measurable set $T$, then

$$
\int N(f, T, y) d \Phi y=\int_{T} J f(x) d x .
$$

Proof. Take $\bar{x}^{1}=(1,0) ; \bar{x}^{2}=(0,1) ; \bar{x}^{3}=(-1,0)$ for $x \in T$ and apply 6.1.

7. Functions on the line to the line.

7.1 Definition. If $f$ is a function on $E_{1}$ to $E_{1}$, then $D+f$ is the function such that

$$
D^{+} f(x)=\limsup _{z \rightarrow x+} \frac{f(z)-f(x)}{z-x} .
$$

7.2 TheOREM. If $f$ is a Lebesgue measurable function on $E_{1}$ to $E_{1}, T$ is a Lebesgue measurable set of numbers and 
then

$$
\left|D^{+} f(x)\right|<\infty \text { for } x \in T \text {, }
$$

$$
\int N(f, T, y) d y=\int_{T}\left|D^{+} f(x)\right| d x .
$$

Proof. From Theorem 9.9 of Saks $\left({ }^{14}\right)$, chap. 9, we infer the existence of a set $S \subset T$ such that

$$
|T-S|=0, \quad J f(x)=\left|D^{+} f(x)\right| \text { for } x \in S .
$$

Hence Theorem 5.2 of this paper yields

$$
\int N(f, S, y) d y=\int_{S} J f(x) d x=\int_{S}\left|D^{+} f(x)\right| d x=\int_{T}\left|D^{+} f(x)\right| d x .
$$

But Theorem 4.6 of Saks $\left({ }^{14}\right)$, chap. 9, tells us

$$
\left|f^{*}(T-S)\right|=0
$$

and we complete the proof by observing that the last relation implies $N(f, S, y)=N(f, T, y)$ for almost all numbers $y$.

(14) Loc. cit. (7).

University of California,

Berkeley, Calit. 